\title{
PRIMARY MUCINOUS ADENOCARCINOMA OF RENAL PELVIS IN SOLITARY PELVIC KIDNEY
}

\author{
JULIAN WAN, M.D. \\ DANA A. OHL, M.D. \\ LEE WEATHERBEE, M.D.
}

From the Departments of Surgery (Section of Urology) and Pathology, University of Michigan Medical Center, and Ann Arbor Veterans Affairs Medical Center, Ann Arbor, Michigan

\begin{abstract}
Mucinous adenocarcinoma of the renal pelvis is a rare tumor. We report the case of a fifty-three-year-old man with this unusual cancer and review the literature. Usual features of this tumor include long duration of symptoms, an association with calculi and hydronephrosis, and a preoperative appearance of an inflammatory condition.
\end{abstract}

Nearly 90 percent of all carcinomas of the renal pelvis are transitional cell carcinomas. Of the remaining 10 percent, most are squamous cell carcinomas. ${ }^{1}$ In contrast, the mucinous adenocarcinoma of the renal pelvis is an uncommon primary tumor. Review of the English urologic literature shows less than 40 cases. $^{2-11}$ Because this tumor is so infrequent, a preoperative diagnosis is rarely made. We report an occurrence in a solitary pelvic kidney where the diagnosis was made only on pathologic analysis, and we review the literature.

\section{Case Report}

A sixty-three-year-old man had a solitary left pelvic kidney and a history of recurrent episodes of pyelonephritis and renal calculi. He presented to another hospital with a threemonth history of intermittent fevers, chills, fatigue, and lower abdominal discomfort. Laboratory studies found a white blood cell count of 5.0 and a serum creatinine of $9.1 \mathrm{mg}$ percent despite a baseline serum creatinine of $3.0 \mathrm{mg}$ percent. An attempt was made to pass a ureteral stent without success, and he was then transferred to the Ann Arbor VA Medical Center. Following hemodialysis, the serum creatinine level came down to $3.5 \mathrm{mg}$ percent, and a subsequent cystoscopic examination and retrograde stent placement were successful. Hydronephrotic calices with an ectatic ureter were seen on retrograde pyelogram (Fig. 1). Urine collected from the renal pelvis grew out Proteus mirabilis and Candida albicans. Systemic antibiotic and antifungal therapy was given.

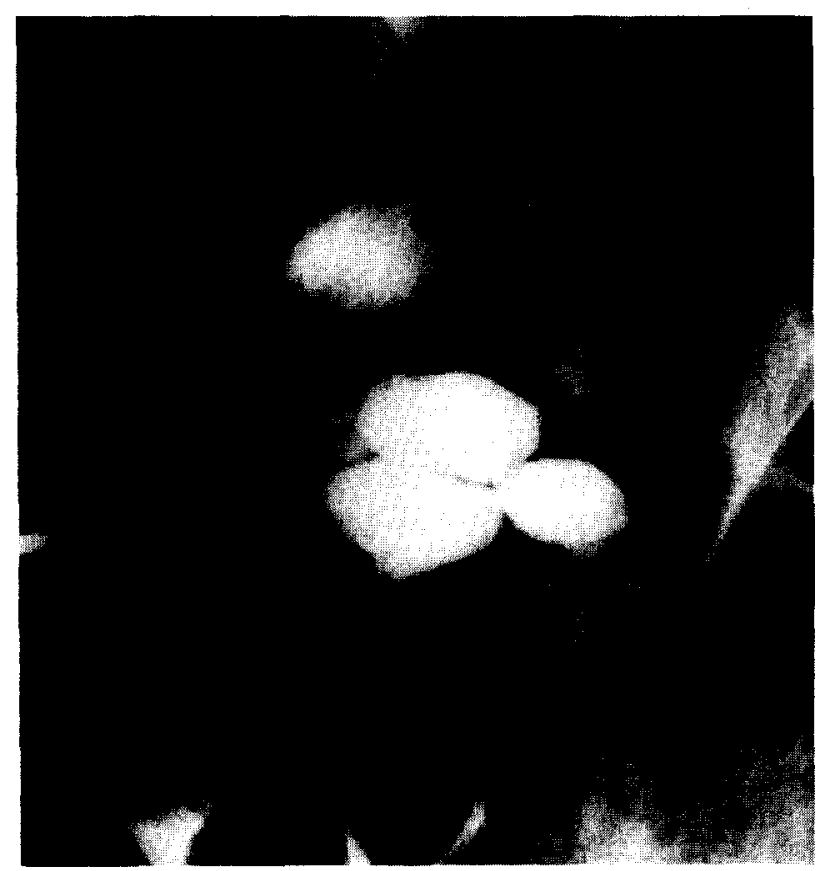

Figure 1. Retrograde pyelogram showing hydronephrotic pelvic kidney. 


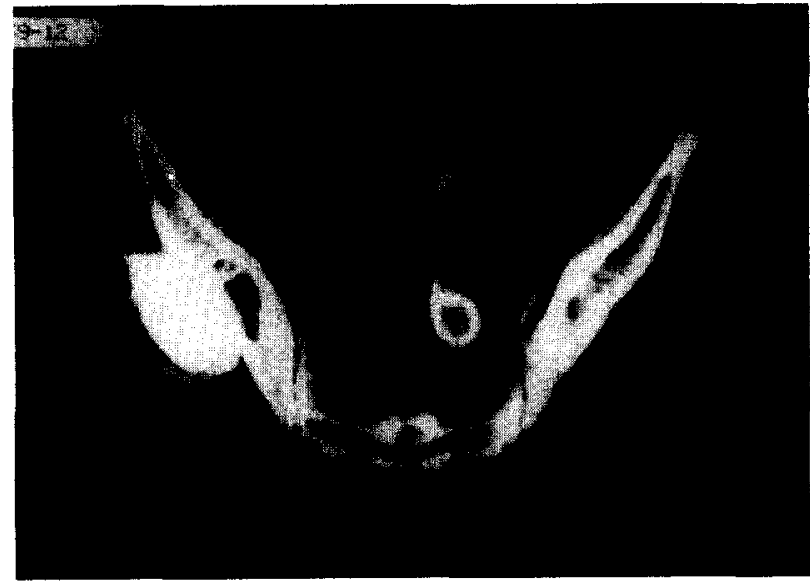

FIgURE 2. Non-contrast CT scan of pelvis showing stent within kidney.

After two weeks of treatment without abatement of the fevers and abdominal discomfort, a non-contrast computerized tomography (CT) scan was performed (Fig. 2). It showed a pelvic kidney with a possible renal abscess. Because of the lack of progress the kidney was explored surgically. It was found to be irregular and nodular with little normal-appearing parenchyma. Purulent exudate filled the pelvis and ureter. Because of its poor condition, the kidney along with the ureter were removed. Pathologic examination of the specimen revealed an invasive mucinous adenocarcinoma of the renal pelvis.

Postoperatively the patient defervesced and continued on hemodialysis with a stable creatinine level of 3.5 to $4.0 \mathrm{mg}$ percent. It was recommended that with the finding of a positive margin that external beam radiation should be given to the surgical bed. The patient, however, later declined further treatment. The patient succumbed to a rapidly progressive sepsis of unknown etiology one and a half years after surgery. Postmortem examination was refused.

\section{Pathologic findings}

The removed kidney was irregular and nodular in appearance. It measured $10 \times 7 \times 6 \mathrm{~cm}$ and sectioning revealed extensive fibrosis and hemorrhage with little recognizable parenchyma. Yellow purulent material filled the calices and the ureter (Fig. 3). A hard, black 5$\mathrm{mm}$ calculus was found in the pelvis.

Microscopic examination revealed an invasive mucin-producing adenocarcinoma (Fig. 4). The carcinoma arose from within the pelvic transitional mucosal epithelium and spread into the collecting ducts. Infiltration through the

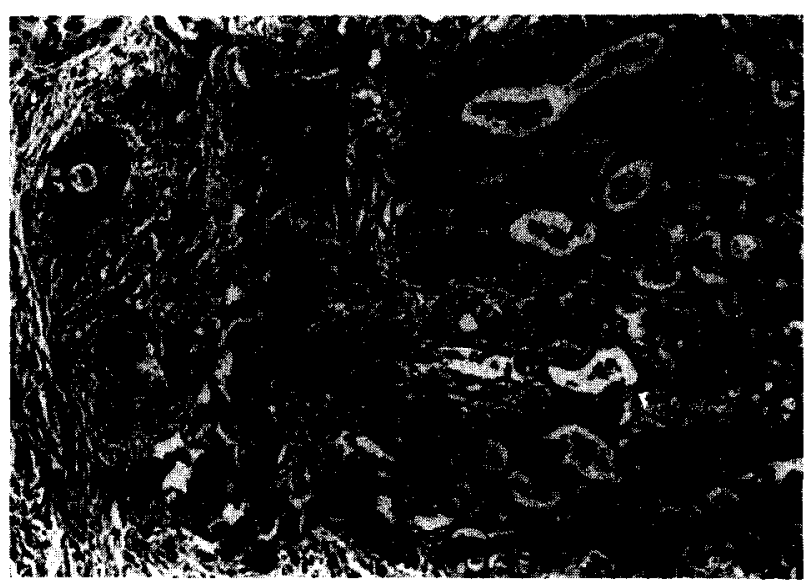

FIGURE 3. Irregular nodular hydronephrotic specimen filled with exudate.

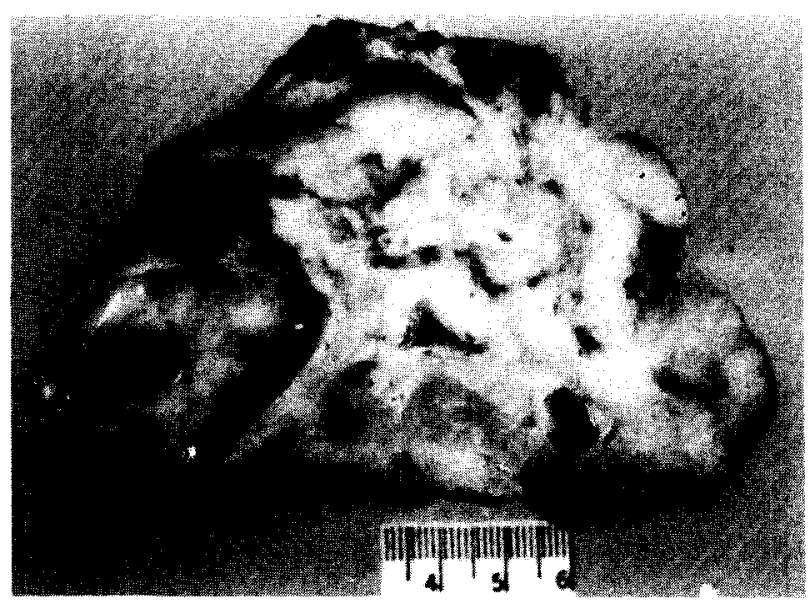

Figure 4. Photomicrograph of mucinous adenocarcinoma showing attempts at gland formation and mucin within lumens.

parenchyma was noted. Invasion of the muscularis and intima of the arteries and within some of the perineural lymphatics was also seen. Throughout the kidney there was extensive necrosis and interstitial fibrosis. The carcinoma extended to the renal capsule along one margin, while the ureter and surrounding adipose tissue were free.

\section{Comment}

Mucinous adenocarcinoma of the renal peivis is a rare primary tumor. The majority of the reported cases occurred in patients over fifty years of age with only a few under the age of forty. ${ }^{2,3,11}$ No difference in prevalence has been noted between the sexes. ${ }^{3,5}$ Though unusual in its presenting in a solitary pelvic kidney, this case illustrates the salient features of this infrequently encountered tumor.

First, chronicity of presenting symptoms is a common finding. Murphy and Stevenson ${ }^{2}$ in 
their review in 1970 found an average duration of symptoms to be about four years. This prolonged duration of symptoms also was noted in a subsequent review by Aufderheide and Streitz in $1973^{3}$ whose 2 case reports each had histories of recurrent infections and stones lasting over two decades.

Second, while infections and renal calculi are often associated together, they are usually not associated with carcinomas. Yet, more than two thirds of the previously reported cases had associated calculi and over three-quarters had related hydronephrosis. ${ }^{2,5}$

Last, nearly all previously reported patients were thought preoperatively to have some benign infectious or inflammatory condition. An abscess, chronic pyelonephritis, or an infected stone were often suspected. ${ }^{2,3}$ The sole exception in the literature is the case of a seventy-four-year-old man with a large right staghorn calculus who presented with gross hematuria. ${ }^{7}$ An intravenous pyclogram in that case was ordered and showed a suspicious upper pole mass. Subsequent angiography demonstrated neovascularity thought to be consistent with renal cell carcinoma.

These observations, while not proving a causative mechanism, do suggest a theory as to how the lining of the renal pelvis, an epithelium which normally lacks any glandular elements, could develop a mucin-secreting tumor. It is proposed that chronic infections and calculi can render the renal pelvis urothelium susceptible to the development of mucin-secreting adenocarcinomas.

This concept is supported by both pathologic and clinical observations. It is known that the urothelium which lines the urinary tract is multi-potential and any chronic irritation can lead to both squamous and glandular metaplasia. ${ }^{12.13}$ It also has been observed that in older patients with bladder exstrophy, a condition which exposes the urothelium to constant environmental trauma and irritation, that metaplasia to a glandular mucus-secreting epithelium occurs. ${ }^{4,8}$ Significantly, the most common bladder tumor in these patients is the mucinous adenocarcinoma. ${ }^{1}$ Thus, it is thought that under the influence of the chronic irritation of recurrent infections and/or calculi, metaplasia occurs which could lead to the formation of mucinous adenocarcinomas. $5,12,13$

Data concerning survival are difficult to interpret accurately given the small number of cases. From the available data gathered from the published reviews, the prognosis appears to be poor, with about half of the patients dying within two years of surgery. ${ }^{2.4}$

Though seldom seen, the possibility of this tumor should be recognized when treating any patient with recurrent stones and infections. This is particularly important if any sort of open renal surgery is contemplated. As there are reports of recurrence at the site of surgical spillage and retained ureteral tissue, and the fact that this tumor is usually not suspected preoperatively, some clinicians have recommended that special care be taken in handling cases involving chronic stones and/or infections, especially if mucin is found in the collecting system. ${ }^{3.5}$ The gross appearance of the tumor may help alert the surgeon and adequate precautions against spillage can be taken. Radical nephrectomy and complete removal of the ureter remains the preferred surgical treatment and should be performed in any suspected cases.

\section{Section of Urology \\ The University of Michigan 2918G Taubman Center \\ Box 0330 \\ Ann Arbor, Michigan 48109}

(DR. OHL)

\section{References}

1. Mostofi FK, Davis CJ Jr, and Sesterhenn IA: Pathology of tumors of the urinary tract, in Skinner DG, and Lieskovsky F (Eds): Diagnosis and Management of Genitourinary Cancer, Philadelphia, W.B. Saunders, 1988, chap 7, pp 83-117.

2. Murphy T, and Stevenson JE: Primary adenocarcinoma of the renal pelvis: report of a case, J Urol 104: 62 (1970).

3. Aufderhcide A, and Streitz JM: Mucinous adenocarcinoma of the renal pelvis: report of two cases, Cancer 33: 167 (1973)

4. Aguilo JJ, and Furlow WL: Mucus-producing adenocarcinoma of renal pelvis, Urology 4: 488 (1974).

5. Liwnicz BH, et al: Mucinous adenocarcinoma of the renal pelvis: discussion of possible pathogenesis, J Urol 114: 306 (1975)

6. Kutscher HA, Trainer TD, and Fagan WT: Mucinous adenocarcinoma of renal pelvis, Urology 20: 94 (1982).

7. Wild RM, Ladaga LE, Copeland JS, and Schellhammer PF: Primary mucus-secreting adenocarcinoma of the renal pelvis, South Med J 72: 1343 (1979).

8. Joshi K, Jain K, Mathur S, and Mehrotra GC: Mucinous adenocarcinoma of the renal pelvis, Postgrad Med J 56: 442 (1980).

9. Mirone V, Prezioso D, Palombini S, and Lotti T: Mucinous adenocarcinoma of the renal pelvis, Eur Urol 10: 284 (1984).

10. Jain BJ: Adenocarcinoma of the renal pelvis, J Urol 97: 55 (1967).

11. Brawer MK, and Waisman J: Mucinous adenocarcinoma probably arising in the renal pelvis and ureter: a case report, J Urol 123: 424 (1980).

12. Mostofi FK: Potentialities of bladder epithelium, J Urol 71: 705 (1954).

13. MacLean JT, and Fowler VR: Pathology of tumors of the renal pelvis and ureter, J Urol 75: 384 (1956). 